\title{
Chandra Observations of WR 147 Reveal a Double X-ray Source
}

\author{
Svetozar A. Zhekov ${ }^{1,3}$ and Sangwook Park ${ }^{2}$
}

\begin{abstract}
We report the first results from deep X-ray observations of the Wolf-Rayet binary system WR 147 with the Chandra HETG. Analysis of the zeroth order data reveals that WR 147 is a double X-ray source. The northern counterpart is likely associated with the colliding wind region, while the southern component is certainly identified with the WN star in this massive binary. The latter is the source of high energy X-rays (including the Fe $\mathrm{K} \alpha$ complex at $6.67 \mathrm{keV}$ ) whose production mechanism is yet unclear. For the first time, X-rays are observed directly from a WR star in a binary system.
\end{abstract}

Subject headings: stars: individual (WR 147 ) — stars: Wolf-Rayet — X-rays: stars - shock waves

\section{To Appear in The Astrophysical Journal Letters}

\section{Introduction}

The Wolf-Rayet (WR) star WR 147 is a massive binary system that is bright in X-rays and is a composite radio source. Being the second closest WR star known allowed its emission to be spatially resolved in the radio, near infrared (NIR) and optical. High-resolution radio observations showed that its southern component, WR 147S (the WN8 component in the binary), is a thermal source while its northern counterpart, WR $147 \mathrm{~N}$, is a non-thermal source (Abbott et al. 1986; Moran et al. 1989; Churchwell et al. 1992; Contreras et al. 1996; Williams et al. 1997; Skinner et al. 1999). High-resolution NIR data revealed two sources separated by $\approx 0$.'64 and WR $147 \mathrm{~N}$ was classified as a B0.5V star (Williams et al. 1997). An earlier spectral class, O8-O9 V-III, was suggested from Hubble Space Telescope observations which spatially resolved the binary components (Niemela et al. 1998). At a distance

\footnotetext{
${ }^{1}$ JILA, University of Colorado, Boulder, CO 80309-0440, USA; zhekovs@colorado.edu

${ }^{2}$ Department of Astronomy and Astrophysics, Pennsylvania State University, 525 Davey Laboratory, University Park, PA 16802, USA; park@astro.psu.edu

${ }^{3}$ On leave from Space Research Institute, Sofia, Bulgaria
} 
of $630 \pm 70$ pc to WR 147 (Churchwell et al. 1992), the projected (or minimum) binary separation is $403 \pm 13$ au. Since the binary components are massive stars, the interaction of their strong winds should form a colliding-stellar wind (CSW) region (Prilutskii \& Usov 1976; Cherepashchuk 1976). Interestingly, from the analysis of radio and IR images, Williams et al. (1997) concluded that the WR $147 \mathrm{~N}$ radio peak is displaced by $0^{\prime \prime} .07$ southward from its IR counterpart. This value is within the uncertainties of the data but if it is real, the non-thermal radio source can be associated with the CSW region located in vicinity of the OB companion. The CSW scenario is supported by the analysis of the Chandra High Resolution Camera (HRC) image (although with a very limited photon statistics and having no spectral resolution) which concluded that the X-ray emission is spatially extended and it peaks north of the WN8 star although a deeper X-ray image is needed to accurately determine the degree of spatial extension (Pittard et al. 2002). It is worth noting that contribution from the massive stars in the binary cannot be excluded since these objects are X-ray sources themselves (e.g. Güdel \& Nazé 2009 and the references therein).

X-ray observations are very important for studying CSWs. They provide us with direct information about the physical conditions in the hot plasma behind strong shocks, which are likely the place where non-thermal radio emission forms. The earlier X-ray observations of WR 147 have revealed the presence of thermal emission from high temperature plasma (Caillault et al. 1985; Skinner et al. 1999), but the CSW plasma characteristics were more tightly constrained only from the higher signal-to-noise XMM-Newton data (Skinner et al. 2007). Skinner et al. reported a detection of the Fe K $\alpha$ complex at $6.67 \mathrm{keV}$ and their analysis showed that the plasma temperature is higher than the maximum temperature in the colliding-wind shock. Consequently, Zhekov (2007) modeled the XMM-Newton spectra successfully in the framework of the CSW scenario but only at the requirement the stellar wind velocities were by a factor of $1.4-1.6$ higher than their currently accepted values.

The fact that WR 147 is a wide binary at a relatively small distance gives us an opportunity to examine the CSW phenomenon in more detail that is to carry on spatially resolved X-ray studies. This motivated our deep observations with the Chandra High Energy Transmission Gratings (HETG). In this Letter we report the first part of our analysis (based on the zeroth order data) which reveals a double X-ray source in WR 147 .

\section{Observations and Data Reduction}

WR 147 was observed with Chandra in the period Mar 28 - Apr 10, 2009. The observations in configuration HETG-ACIS-S were carried out in eight occasions (Chandra ObsIds: 9941, 9942, 10675, 10676, 10677, 10678, 10893 and 10897) that provided 7,300 zeroth order 
counts in a total effective exposure of $286 \mathrm{ksec}$. The CIAO 4.1.2 1 data analysis software was used through this study in combination with the Chandra calibration database CALDB v.4.1.3. Following the CIAO Science Threads, the original or combined event files were used for determining the source coordinates, source variability and spectral extractions. By default, the pixel randomization is switched off in grating data.

\section{Analysis and Results}

To have a full advantage of the total source counts in our analysis of the zeroth order data, it is necessary to combine all data sets. As a prerequisite to this process, it is important to estimate the accuracy of the source coordinates in the resultant total image. This was done in two steps.

First, the CIAO command wavdetect was run on each of the eight observations and an average position of the source was calculated. The errors of the mean were propagated from the individual errors derived from wavdetect. Second, all the zeroth order data were merged eight times (CIAO command merge_all) with a different reference image. The source coordinates were determined for each merged image by running wavdetect. The maximum difference in the source coordinates from one merged image to another was 0.002 and 0.02 , correspondingly for the right ascension and declination. The source positions are given in Table 1. We note that the center of the source in the merged data is practically the same as the one determined from averaging coordinates of the source centers derived for each individual observation. All this makes us confident that our analysis is not influenced by the choice of the reference image. The derived X-ray coordinates of WR 147 are in good agreement (within the $1 \sigma$ of the Chandra positional accuracy 2) with the ones determined for this object in the optical (HST Guide Star Catalog) and in the radio (Table 1).

\subsection{Images}

The considerably better photon statistics $(7,300 \mathrm{cts})$ of the total zeroth order HETG image compared to that of the previous Chandra HRC image ( 148 cts, Pittard et al. 2002) gave us an opportunity to study in detail the spatial extent of the X-ray emission from this object. It also allowed us to carry on image analysis in different photon energy bands.

\footnotetext{
${ }^{1}$ Chandra Interactive Analysis of Observations (CIAO), http://cxc.harvard.edu/ciao/

${ }^{2}$ http://acs.harvard.edu/proposer/POG: $§ 5.4 .1$
} 
The relatively small spatial scale of the WR 147 emission in the radio and optical $(0 \prime .57-0.64$ which corresponds to $\sim 1.2-1.3$ ACIS pixels) prompted the use of subpixel images and image deconvolution techniques. We applied a deconvolution procedure, based on a maximum likelihood method (Richardson 1972; Lucy 1974), that was successfully used in the image analysis of SNR 1987A, an object with a small spatial extent of $\sim$ 1".6 (Burrows et al. 2000; Park et al. 2002, 2004, 2006; Racusin et al. 2009). The main result is that the morphology of the X-ray emission of WR 147 changes in different energy bands: its northern part is brightest in the (1.0-2.0 keV) range and gradually weakens at higher energies. This likely indicates that a soft X-ray source is located there while most of the hard energy photons originate in the southern part of WR 147 . This pattern is clearly seen from the raw subpixel X-ray images as well as from the deconvolved ones (Fig. 1) and is also illustrated by the sources relative brightness derived from direct photon counting (see lower right panel in Fig. 3). It is thus conclusive that WR 147 is a double X-ray source, consisting of a northern (WR 147N ) and a southern (WR 147S ) part with different spectral characteristics. A separation of $\approx 0$ "' 60 between the positions of maximum emission of WR $147 \mathrm{~N}$ and WR $147 \mathrm{~S}$ is directly measured from the deconvolved $(1.0-2.0 \mathrm{keV})$ image.

The existence of a spatial separation between WR 147N and WR 147S gets additional support from measuring the WR 147 coordinates in different energy bands. We ran the CIAO command wavdetect on the eight merged images. We see that there is an offset of $\approx 0.51$ between the source coordinates in the soft $(1.0-2.0 \mathrm{keV})$ and hard $(6.0-8.0 \mathrm{keV})$ energy bands (Table 1). Interestingly, the source coordinates in the latter almost perfectly coincide with the ones in the optical and radio. It is thus conclusive that the X-ray hard-photon emitter (WR 147S ) is in fact the WR star itself (a WN8 object). Furthermore, a direct comparison of the WR 147 images in the radid 3 and X-rays reveals a nice correspondence between the locations of the radio and X-ray WR $147 \mathrm{~N}$ sources (Fig. 1) which is suggestive that this is the CSW region in this binary system.

\subsection{Undispersed Spectra}

Guided by the results from the image analysis, we extracted the X-ray spectra of WR 147 , WR 147N and WR 147S from the total (merged) zeroth order data by using the CIAO script specextract (for extraction regions see Fig. 2). We fitted the spectra with standard as well as custom models in version 11.3.2 of XSPEC (Arnaud 1996). We note that

\footnotetext{
${ }^{3}$ The radio data are from the NRAO Archive. The National Radio Astronomy Observatory is a facility of the National Science Foundation operated under cooperative agreement by Associated Universities, Inc.
} 
due to the small spatial separation between WR 147N and WR 147S their spectra are not completely disentangled from each other. In our upcoming analysis of the first-order data, we will consider a more elaborated modeling of the undispersed spectra in conjunction with the dispersed ones. We adopted a simpler approach here which is nevertheless instructive for the emerging physical picture.

As seen from Fig. 3, the total spectrum of WR 147 is very similar to that obtained with XMM-Newton (Skinner et al. 2007): it is heavily absorbed, having most of its X-ray counts at energies $\mathrm{E} \geq 1 \mathrm{keV}$. For consistency with the previous studies, we adopted the same set of WN abundances (see Skinner et al. 2007; Zhekov 2007). A simple 1T shock model (vpshock in XSPEC) gave a similar quality of the fit as for the XMM-Newton spectra of WR 147 . The X-ray absorption and the plasma temperature (with $90 \%$ confidence intervals in brackets) were $\mathrm{N}_{H}=2.5[2.3-2.9] \times 10^{22} \mathrm{~cm}^{-2}, \mathrm{kT}=2.9[2.3-3.2] \mathrm{keV}$, and the derived abundances fell within the $90 \%$ confidence intervals of the XMM-Newton spectral fits (Skinner et al. 2007). The total observed flux was $\mathrm{F}_{X}(0.5-10 \mathrm{keV})=1.28 \times 10^{-12} \mathrm{erg} \mathrm{s}^{-1}$, about $15 \%$ smaller than that from the XMM-Newton spectra. A better constraint on this difference would be possible after completing upcoming analysis of the first-order HETG data.

Exploring the physical picture that identifies WR $147 \mathrm{~N}$ with the CSW region in the binary system, the X-ray spectra of WR $147 \mathrm{~N}$ and WR $147 \mathrm{~S}$ were fitted simultaneously and they shared the same WN abundances set. The latter is justified by that the shocked WN wind dominates the X-ray emission of the CSW region (Zhekov 2007; for the use of discrete temperature models see $\S 5.2$ therein). The $1 \mathrm{~T}$ shock model with individual postshock temperatures for WR $147 \mathrm{~N}$ and WR $147 \mathrm{~S}$ and a common X-ray absorption gave a poor quality of the fit (reduced $\chi^{2} \approx 1.5$ ). An acceptable fit was obtained if the spectra had individual X-ray absorption (see Fig. 3 and Table 2). It should be emphasized that in both sources the plasma temperature well exceeds $1 \mathrm{keV}$.

As in the image analysis, we see that the northern source is softer than its southern counterpart. Also, the latter (the WN8 star) is the place where the Fe K $\alpha$ complex at $6.67 \mathrm{keV}$ comes from (see the inset in Fig. 3). WR 147S is subject to an excess X-ray absorption compared to WR $147 \mathrm{~N}$. Applying the Gorenstein (1975) conversion (NH = $2.22 \times 10^{21} \mathrm{~A}_{V} \mathrm{~cm}^{-2}$ ), the absorption towards WR $147 \mathrm{~N}$ almost perfectly corresponds to the optical extinction of WR $147\left(\mathrm{~A}_{v}=11.6 \mathrm{mag}, \mathrm{A}_{V}=\mathrm{A}_{v} / 1.11\right.$; van der Hucht 2001).

We thus note that the results from image and spectral analysis seem consistent with a physical picture where WR $147 \mathrm{~N}$ resides in the CSW region of the binary, while the X-ray source WR $147 \mathrm{~S}$ is likely located deeper in the WR wind. 


\subsection{MARX Simulations}

We used version 4.4 of the MARX 4 software to simulate the observational situation with WR 147 . We ran a 286-ksec HETG exposure for a X-ray source composed of two point sources with spectral characteristics corresponding to those of WR 147N and WR 147S (Table 2). The sources were located at the positions of maximum emission for the northern and southern sources as in the (1.0-2.0 keV) deconvolved image of WR 147 . We note a nice correspondence between the simulated and observed data (Fig. 1).

All this makes us more confident about the results from our analysis, that illustrate the superior capabilities of the Chandra observatory even when working on their very edge.

\section{Discussion}

Analysis of the zeroth order HETG data showed that Chandra resolved WR 147 into a double X-ray source. Its two counterparts, WR 147N and WR 147S, are most likely identified correspondingly with the CSW region and the WN8 star in this wide binary system. We recall that the maximum temperature in the CSW region is determined by the terminal wind velocities in the binary and for WR 147 their currently accepted values could not provide the plasma temperature deduced from the X-ray spectra (Skinner et al. 2007). This is why, successful CSW models of WR 147 were possible only for wind velocities being a factor of $1.4-1.6$ higher (Zhekov 2007). We note that all this is based on analysis of the unresolved $X M M-N e w t o n$ data which assumed that the CSW region is responsible for the total X-ray emission from WR 147 . The high resolution Chandra data resolve this discrepancy by revealing two sources with different spectral characteristics.

We used the CSW model with the nominal stellar wind parameters $\left(\mathrm{V}_{W R}=950 \mathrm{~km} \mathrm{~s}^{-1}\right.$, $\dot{M}_{W R}=4 \times 10^{-5} \mathrm{M}_{\odot} \mathrm{yr}^{-1} ; \mathrm{V}_{O}=1600 \mathrm{~km} \mathrm{~s}^{-1}, \dot{M}_{O}=6.6 \times 10^{-7} \mathrm{M}_{\odot} \mathrm{yr}^{-1} ;\left[\dot{M}_{O} V_{O} / \dot{M}_{W R} V_{W R}\right]=$ 0.028; for CSW model details see Zhekov 2007) to fit the X-ray spectrum of WR $147 \mathrm{~N}$. As seen from Fig. 3, the shape of the observed spectrum is perfectly matched, that is there is NO temperature discrepancy between the model and observations any more. But, the model overestimates the total luminosity of WR $147 \mathrm{~N}$ by a factor of $\sim 16$. We note that this discrepancy is in general trackable. The CSW X-ray luminosity scales with the mass loss rate $\dot{M}$, wind velocity $v$ and binary separation as $L_{X} \propto \dot{M}^{2} v^{-3} D^{-1}$ (Luo et al. 1990; Myasnikov \& Zhekov 1993). If the mass-loss rates are factor of $\sim 2$ or even more lower than assumed because of their intrinsic uncertainties or because the winds are clumped (e.g.

\footnotetext{
${ }^{4}$ See http://space.mit.edu/CXC/MARX/
} 
Crowther 2007), this can account for most of the luminosity mismatch, and the unknown orbital inclination (larger binary separation) would account for the difference. All this will be explored in detail in our upcoming combined modeling of the first-order spectra and the undisperesed ones.

While this gives more confidence that WR 147N resides in the CSW region of the binary system, an identification of WR $147 \mathrm{~N}$ with the OB star in the system cannot be completely ruled out. Our analysis of the X-ray spectral lines (from the first-order HETG spectra) will be crucial in this respect. For example, if suppressed forbidden line in the He-like triplets is found (an indication of high density or strong UV emission), it will favor the OB star origin for WR $147 \mathrm{~N}$. Unfortunately, the luminosity of the OB companion is poorly constrained (Lèpine et al. 2001) which prevents estimating its contribution to the X-ray emission of WR $147 \mathrm{~N}$ based on the $\mathrm{L}_{X}-\mathrm{L}_{b o l}$ relation. But, the OB stars are in general soft X-ray sources with plasma temperatures less than $1 \mathrm{keV}$ (see $\S 4.1 .2$ and $\S 4.3$ in the review paper of Güdel \& Nazé 2009). Given the high X-ray absorption to WR 147 (§3.2), we thus anticipate that this contribution will not be appreciable unless the OB star in WR 147 is a rare hot magnetic object (see $\S 4.6$ in Güdel \& Nazé 2009).

On the other hand, the fact that the WN8 star is a hard-energy X-ray source (WR 147S ) is indeed puzzling. Skinner et al. (2002a,b) have discussed in some detail possible mechanisms for X-ray production in WN stars when the high-energy tail was established with certainty in the X-ray emission from presumably single WR stars: WR 6 and WR 110. These mechanisms include: instability-driven wind shocks (IDWS); magnetically confined wind shocks (MCWS); wind accretion shocks; colliding wind shocks (including the case of stellar wind shocking onto a close companion); non-thermal X-ray emission. We note that none of these mechanisms finds solid observational support for the moment and each of them has its own limitations and caveats. For example, the IDWS should be considerably soft X-ray emitters while the MCWS might be able to provide the high plasma temperature observed in WR 147S . But for the massive stellar winds in WRs, this requires a relatively strong global magnetic field in the star itself. At present, such fields have not been reported for Wolf-Rayet stars. Presence of a strong magnetic field may also suggest flare activity but no short-term variability is detected in any of the eight HETG observations (the CIAO tool glvary returns a variability index of zero). The same was found in earlier X-ray observations of WR 147 (Skinner et al. 1999; Skinner et al. 2007). Presence of a compact (or a normal star) companion is intriguing but this may suggest that we have quite a rare opportunity to observe a triple system that initially consisted of at least two or even three massive stars and in the latter case the most massive one has already exploded.

Finally, although the mechanism responsible for the high-energy X-ray production is 
unclear it may not be unique to WR 147 . Apart from earlier detections (WR 6 and WR 110), Skinner et al. (2009) report a hard-energy tail in the X-ray spectra of several more, presumably single, WN stars. It is worth noting that all these WNs have a subtype different from WR 147 while a star of its subtype, WR 40 (a WN8 star), was not detected with XMM-Newton (Gosset et al. 2005). Hopefully, future observations will facilitate solving the mystery of this puzzling phenomenon: how common the high-energy tail is for single WR stars and how it correlates with their subtype. But, WR 147 is unique in the sense that for the first time we detect X-rays directly from a WR star in a binary system. Moreover, the upcoming analysis of the first-order HETG spectra will give us a chance to measure

line parameters (widths, centroids, fluxes) in the X-ray spectrum intrinsic to a WR star (WR 147S ), thus, to study its X-ray plasma characteristics in detail.

This work was supported by NASA through Chandra grant GO9-0013A to the University of Colorado at Boulder, and through grant G09-0013B to the Pennsylvania State University.

Facilities: CXO (HETG, ACIS).

\section{REFERENCES}

Abbott D.C., Bieging J.H., Churchwell E., Torres A.V. 1986, ApJ, 303, 239

Anders E., Grevesse N., 1989, Geochimica et Cosmochimica Acta, 53, 197

Arnaud, K.A. 1996, in Jacoby G., Barnes, J. eds., ASP Conf. Ser. Vol. 101, Astronomical Data Analysis Software and Systems, Astron. Soc. Pac., San Francisco, 17

Burrows, D. N. et al. 2000, ApJ, 543, L149

Caillault, J.-P., Chanan, G. A., Helfand, D. J., Patterson, J., Nousek, J. A., Takalo, L. O., Bothun, G. D., Becker, R. H. 1985, Nature, 313, 376

Cherepashchuk, A.M. 1976, Soviet Astronomy Letters, 2, 138

Churchwell E., Bieging J.H., van der Hucht K.A., Williams P.M., Spoelstra T.A.Th., Abbott D.C. 1992, ApJ, 393, 329

Contreras M.E., Rodriguez L.F., Gomez Y., Velazquez A. 1996 , ApJ, 469, 329

Contreras M.E., Rodriguez L.F. 1999, ApJ, 515, 762

Crowther, P. 2007, ARAA, 45, 177 
Gorenstein, P. 1975, ApJ, 198, 95

Gosset, E., Nazé, Y, Claeskens, J.-F., Rauw, G., Vreux, J.-M., \& Sana, H. 2005, A\&A, 429, 685

Güdel M. \& Nazé Y. 2009, The Astronomy and Astrophysics Review, 17, 309

Lèpine, S., Wallace, D., Shara, M.M., Moffat, A.F.J., \& Niemela, V.S. 2001, AJ, 122, 3407

Lucy, L.B. 1974, AJ, 79, 745

Luo, D., McCray, R., \& MacLow, M.-M. 1990, ApJ, 362, 267

Moran J.P., Davis R.J., Spencer R.E., Bode M.F., Taylor A. R. 1989, Nature, 340, 449

Myasnikov, A.V. \& Zhekov, S.A. 1993, MNRAS, 260, 221

Niemela V.S., Shara M.M., Wallace D.J., Zurek D.R., Moffat A.F.J. 1998, AJ, 115, 2047

Park, S., Burrows, D. N., Garmire, G. P., Nousek, J. A., McCray, R., Michael, E., \& Zhekov, S. A. 2002, ApJ, 567, 314

Park, S., Zhekov, S. A., Burrows, D.N., Garmire, G. P., \& McCray, R. 2004 ApJ, 610, 275

Park, S., Zhekov, S. A., Burrows, D.N., \& McCray, R. 2006, ApJ, 646, 1001

Pittard J.M., Stevens I.R., Williams P.M., et al. 2002, A\&A, 388, 335

Prilutskii, O.F \& Usov, V.V. 1976, Soviet Astronomy, 20, 2

Racusin, J.L., Park, S., Zhekov, S. A., Burrows, D.N., Garmire, G. P., \& McCray, R. 2009, ApJ, 703, 1752

Richardson, W.H. 1972, Opt. Soc. Am., 62, 55

Skinner S.L., Itoh M., Nagase F., Zhekov S.A. 1999, ApJ, 524, 394

Skinner S.L., Zhekov S.A., Güdel M., Schmutz W. 2002a, ApJ, 572, 477

Skinner S.L., Zhekov S.A., Güdel M., Schmutz W. 2002b, ApJ, 579, 764

Skinner S.L., Zhekov S.A., Güdel M., Schmutz W. 2007, MNRAS, 378, 1491

Skinner S.L., Zhekov S.A., Güdel M., Schmutz W., Sokal, K.R. 2009, accepted for publication in AJ, (arXiv:0912.1326) 
van der Hucht, K.A. 2001, New Astronomy Rev., 45, 135

Williams P.M., Dougherty S.M., Davis R.J., van der Hucht K.A., Bode M.F., Setia Gunawan D.Y.A. 1997, MNRAS, 289, 10

Zhekov S.A. 2007, MNRAS, 382, 886 

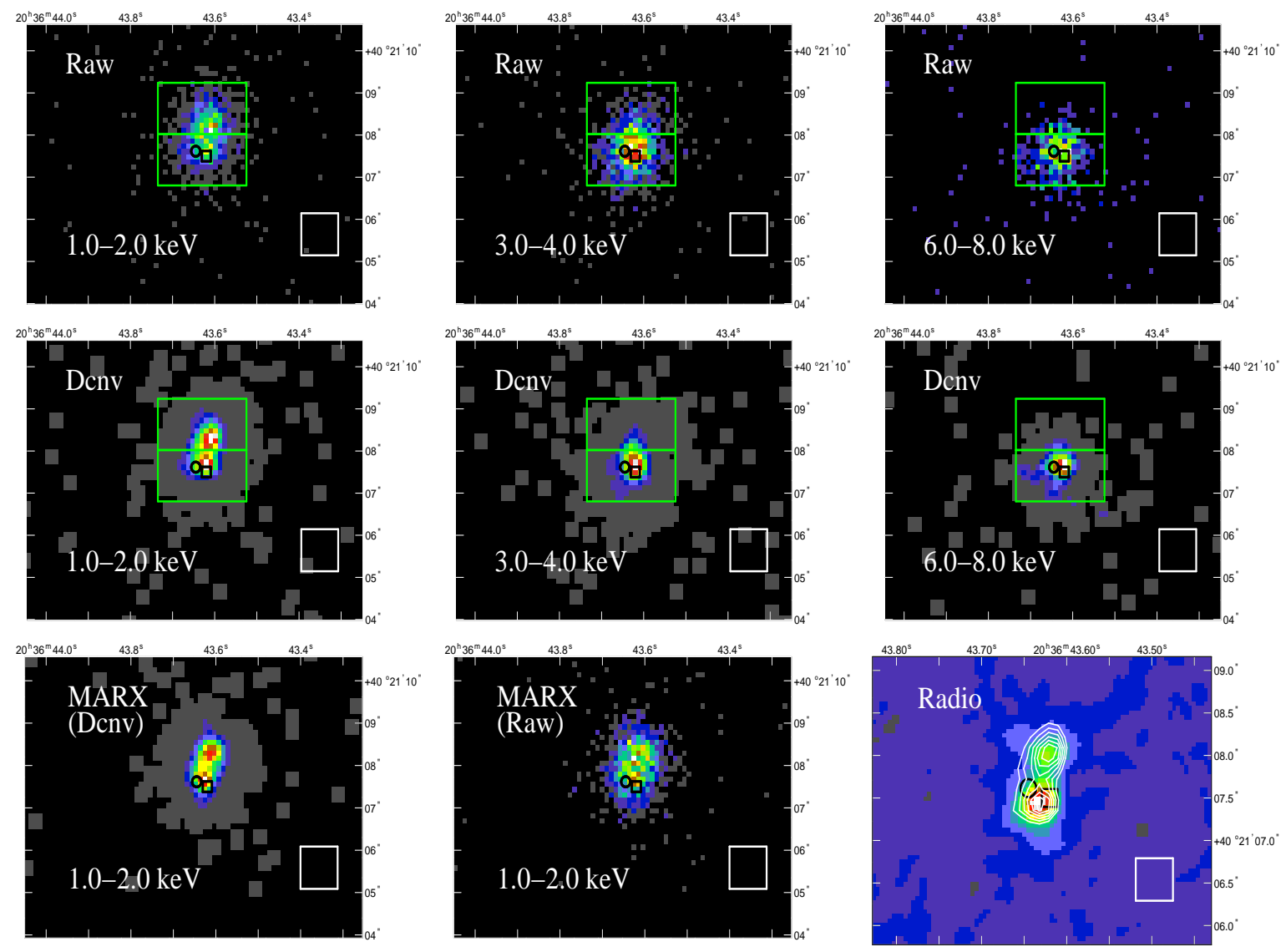

Fig. 1.- Examples of WR 147 X-ray images (pixel size of $0^{\prime \prime} 123$ ) in a linear scale by rows: (1) raw images; (2) deconvolved ones; (3) images from MARX simulations and a radio image. RA(J2000) and Dec(J2000) are on horizontal and vertical axes, respectively. The optical position of WR 147 (HST GSC) is marked by a circle and the position of the southern radio source (Contreras \& Rodriguez 1999) is marked by a square. The scale of each image is illustrated by the $\left(1^{\prime \prime} \times 1^{\prime \prime}\right)$ square in its lower right corner. The 3.6-cm radio image of WR 147 is from a VLA observation on June 28, 1999. The bright southern source is the WN8 star while the fainter emission to its north is the non-thermal source (presumably arising in CSWs). Overlaid are the contours (linearly spaced by 0.1 of the maximum emission) of the deconvolved X-ray image in the (1.0-2.0 keV) energy range. The radio and X-ray images are in relative units normalized to their corresponding maximum brightness. They were aligned that the brightness peak of the southern X-ray source coincided with that of the southern radio source. The region boxes (in green) were used for estimating the relative brightness of WR $147 \mathrm{~N}$ and WR $147 \mathrm{~S}$ (see Fig. 3). Their border line defines how the two sources were separated for the spectral extractions (Fig. 2). 


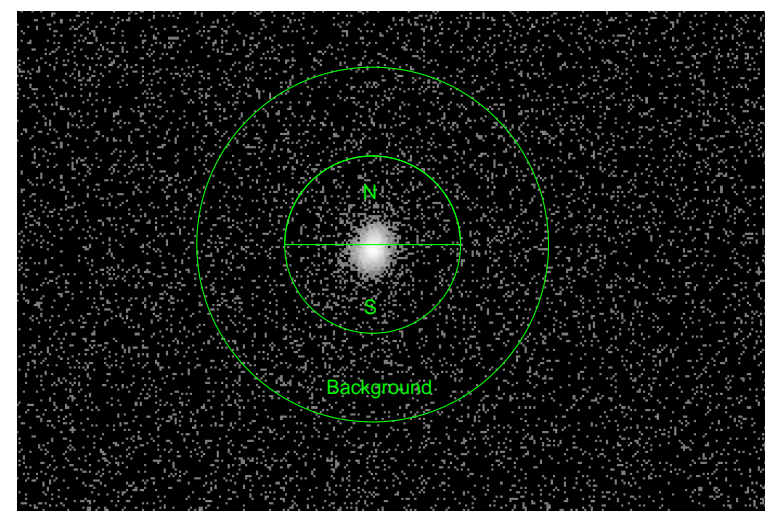

Fig. 2.- The extraction regions for the total WR 147 , WR 147N and WR 147S spectra. 

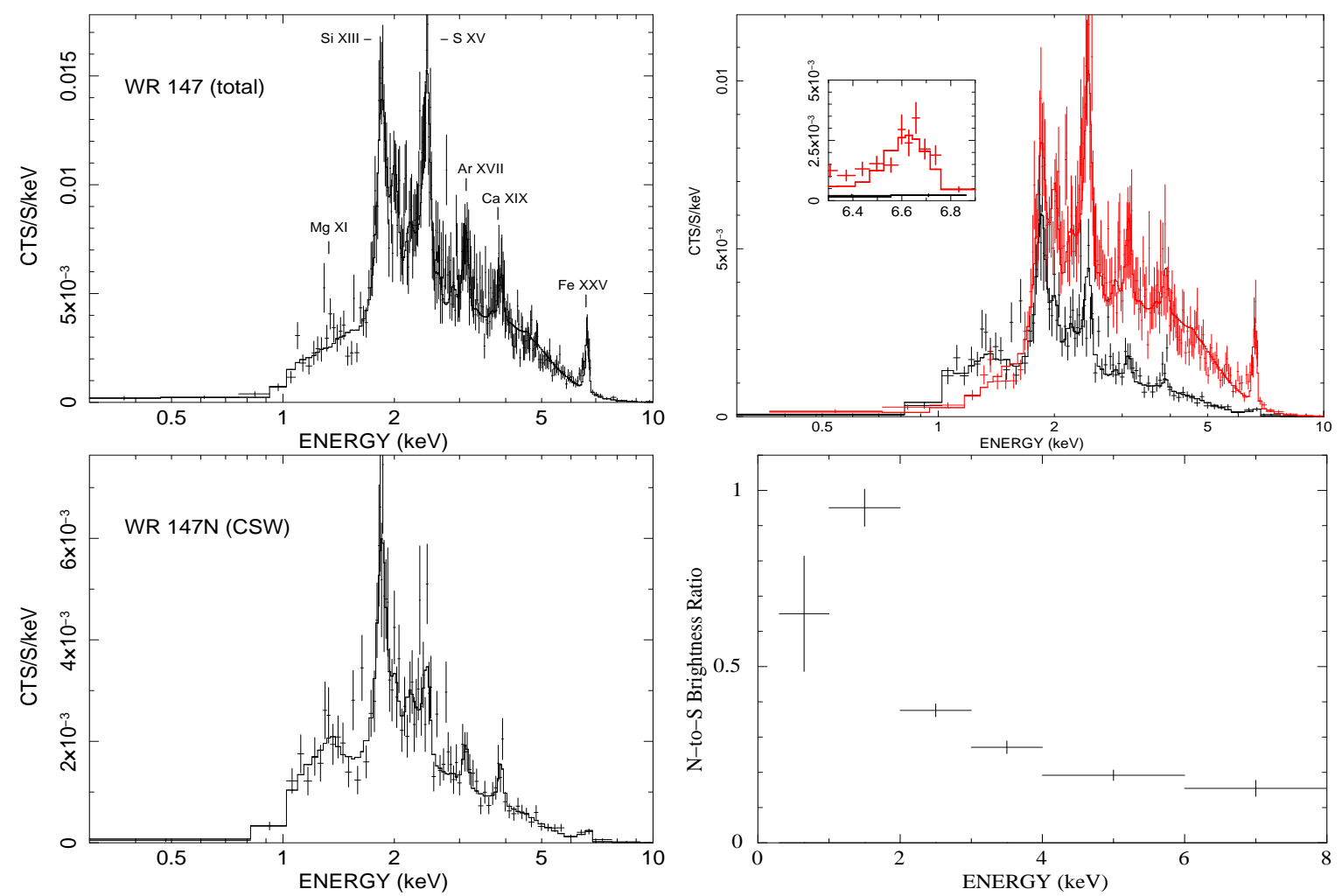

Fig. 3.- WR 147 background-subtracted spectra (rebinned to have a minimum of 20 counts per bin). Upper left panel: The total spectrum overlaid with the best-fit $1 \mathrm{~T}$ shock model $\left(\chi^{2} /\right.$ dof $\left.=269 / 230\right)$. Prominent emission lines are marked. Upper right panel: the WR $147 \mathrm{~N}$ and WR $147 \mathrm{~S}$ (in red) spectra and the $1 \mathrm{~T}$ shock models with individual X-ray absorption. The inset shows the $\mathrm{Fe} \mathrm{K}_{\alpha}$ complex at $6.67 \mathrm{keV}$. Lower left panel: the X-ray spectrum of WR $147 \mathrm{~N}$ overlaid with the CSW model $\left(\chi^{2} /\right.$ dof $\left.=98 / 80\right)$. Lower right panel: The WR 147N -to-WR 147S relative brightness defined in different energy ranges (marked with horizontal bars) by counting the number of photons in the two region boxes as shown in Fig. 1. 
Table 1. WR 147 Positions: X-ray, optical, radio

\begin{tabular}{llll}
\hline \hline & $\begin{array}{c}\alpha_{2000} \\
20^{h} 36^{m}\end{array}$ & $\begin{array}{c}\delta_{2000} \\
+40^{\circ} 21^{\prime}\end{array}$ & $\begin{array}{c}\text { Uncertainty } \\
(\alpha, \delta)\end{array}$ \\
\hline Average & 43.636 & $7^{\prime \prime} .69$ & $\pm 0^{\prime \prime} .02$ \\
Merged & 43.636 & $7^{\prime \prime} .71$ & $\pm 0^{\prime \prime} .01$ \\
$(1.0-2.0 \mathrm{keV})$ & 43.631 & $7^{\prime \prime} .95$ & $\pm 0^{\prime \prime} .03$ \\
$(6.0-8.0 \mathrm{keV})$ & 43.648 & $7^{\prime \prime} .48$ & $\pm 0^{\prime \prime} .02$ \\
HST GSC & 43.64 & $7^{\prime \prime} .62$ & $\pm 0^{\prime \prime} \cdot 32$ \\
Radio $^{a}$ & 43.62 & $7^{\prime \prime} .5$ & $\pm 0^{\prime \prime} .05$ \\
Radio $^{b}$ & 43.64 & $7^{\prime \prime} .5$ & $\pm 0^{\prime \prime} .01$ \\
\hline
\end{tabular}

Note. - The source position by rows: (1) the mean from the eight Chandra observations; (2) from the merged images; $(3,4)$ from 'filtered' merged images; (5) optical; $(6,7)$ radio.

${ }^{\mathrm{a}, b}$ The radio coordinates (Contreras \& Rodriguez 1999; Williams et al. 1997) are for WR 147S (the WN8 star) converted from B1950 into J2000 with the HEASARC coordinate converter (http://heasarc.gsfc.nasa.gov/cgi-bin/Tools/convcoord/convcoord.pl). 
Table 2. 1T Shock Model Results

\begin{tabular}{lcc}
\hline \hline & WR $147 \mathrm{~N}$ & WR $147 \mathrm{~S}$ \\
\hline$\chi^{2} / \mathrm{dof}$ & $309 / 256$ \\
$\mathrm{~N}_{H}\left(10^{22} \mathrm{~cm}^{-2}\right)$ & $2.28[2.08-2.57]$ & $3.83[3.51-4.20]$ \\
$\mathrm{kT}(\mathrm{keV})$ & $1.78[1.52-1.98]$ & $2.36[2.12-2.56]$ \\
$\mathrm{Ne}$ & $19.7[0.0-76.2]$ \\
$\mathrm{Mg}$ & $3.3[0.5-6.7]$ \\
$\mathrm{Si}$ & $5.1[3.9-7.2]$ \\
$\mathrm{S}$ & $6.7[5.5-7.8]$ \\
$\mathrm{Ar}$ & $8.4[5.8-9.8]$ \\
$\mathrm{Ca}$ & $8.3[4.3-12.4]$ \\
$\mathrm{Fe}$ & $10.0[7.7-11.8]$ \\
$\mathrm{F}_{X}{ }^{a}\left(10^{-12} \mathrm{ergs} \mathrm{cm}^{-2} \mathrm{~s}^{-1}\right)$ & $0.324(4.4)$ & $0.921(12.3)$ \\
\hline
\end{tabular}

Note. - Brackets enclose 90\% confidence intervals. All abundances are with respect to their solar values (Anders \& Grevesse 1989). The fixed in the fit abundances are: $\mathrm{H}=1, \mathrm{He}=25.6$, $\mathrm{C}=0.9, \mathrm{~N}=140, \mathrm{O}=0.9$, and $\mathrm{Ni}=1$ (for details see Skinner et al. 2007; Zhekov 2007)

aThe observed X-ray flux $(0.5-10 \mathrm{keV})$ followed in parentheses by the unabsorbed value. 